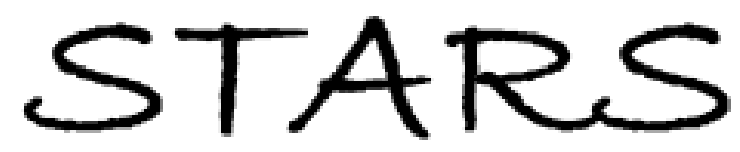

University of Central Florida

STARS

$1-1-2010$

\title{
Space charge limited conduction with exponential trap distribution in reduced graphene oxide sheets
}

\author{
Daeha Joung \\ University of Central Florida
}

A. Chunder

University of Central Florida

Lei Zhai

University of Central Florida

Saiful I. Khondaker

University of Central Florida

Find similar works at: https://stars.library.ucf.edu/facultybib2010

University of Central Florida Libraries http://library.ucf.edu

This Article is brought to you for free and open access by the Faculty Bibliography at STARS. It has been accepted for inclusion in Faculty Bibliography 2010 s by an authorized administrator of STARS. For more information, please contact STARS@ucf.edu.

\section{Recommended Citation}

Joung, Daeha; Chunder, A.; Zhai, Lei; and Khondaker, Saiful I., "Space charge limited conduction with exponential trap distribution in reduced graphene oxide sheets" (2010). Faculty Bibliography 2010s. 318. https://stars.library.ucf.edu/facultybib2010/318

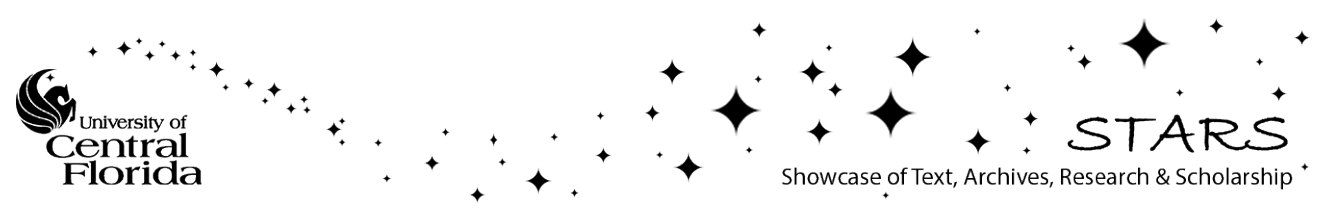




\section{Space charge limited conduction with exponential trap distribution in reduced graphene oxide sheets}

Cite as: Appl. Phys. Lett. 97, 093105 (2010); https://doi.org/10.1063/1.3484956

Submitted: 10 June 2010 . Accepted: 31 July 2010 . Published Online: 01 September 2010

Daeha Joung, A. Chunder, Lei Zhai, and Saiful I. Khondaker

\section{ARTICLES YOU MAY BE INTERESTED IN}

Space-Charge-Limited Currents in Organic Crystals

Journal of Applied Physics 33, 205 (1962); https://doi.org/10.1063/1.1728487

Trap density in conducting organic semiconductors determined from temperature dependence of $\mathrm{J}-\mathrm{V}$ characteristics

Journal of Applied Physics 94, 1283 (2003); https://doi.org/10.1063/1.1582552

Space-charge limited conduction with traps in poly(phenylene vinylene) light emitting diodes

Journal of Applied Physics 82, 6326 (1997); https://doi.org/10.1063/1.366523

\section{Applied Physics Reviews} Now accepting original research 


\title{
Space charge limited conduction with exponential trap distribution in reduced graphene oxide sheets
}

\author{
Daeha Joung, ${ }^{1,2}$ A. Chunder, ${ }^{1,3}$ Lei Zhai, ${ }^{1,3}$ and Saiful I. Khondaker ${ }^{1,2,4, a)}$ \\ ${ }^{1}$ Nanoscience Technology Center, University of Central Florida, Orlando, Florida 32826, USA \\ ${ }^{2}$ Department of Physics, University of Central Florida, Orlando, Florida 32826, USA \\ ${ }^{3}$ Department of Chemistry, University of Central Florida, Orlando, Florida 32826, USA \\ ${ }^{4}$ School of Electrical Engineering and Computer Science, University of Central Florida, Orlando, \\ Florida 32826, USA
}

(Received 10 June 2010; accepted 31 July 2010; published online 1 September 2010)

\begin{abstract}
We elucidate on the low mobility and charge traps of the chemically reduced graphene oxide (RGO) sheets by measuring and analyzing temperature dependent current-voltage characteristics. The RGO sheets were assembled between source and drain electrodes via dielectrophoresis. At low bias voltage the conduction is Ohmic while at high bias voltage and low temperatures the conduction becomes space charge limited with an exponential distribution of traps. We estimate an average trap density of $1.75 \times 10^{16} \mathrm{~cm}^{-3}$. Quantitative information about charge traps will help develop optimization strategies of passivating defects in order to fabricate high quality solution processed graphene devices. () 2010 American Institute of Physics. [doi:10.1063/1.3484956]
\end{abstract}

Nanostructures of graphene oxide (GO) have created a lot of attention as it provides a pathway to produce large quantities of graphene sheets in solution at low cost. ${ }^{1-13}$ The easy processibility of GO and compatibility with various substrates including plastics makes them an attractive candidate for high yield manufacturing of graphene based electronic devices. However, GO is an electrically insulating material consisting of a large number of $\mathrm{C}-\mathrm{O}$ bonds. Removal of $\mathrm{C}-\mathrm{O}$ bonds by chemical and/or thermal reduction technique produces reduced graphene oxide (RGO) sheets and allows one to restore the electrical properties. RGO sheets have been used for field effect transistors (FETs), ${ }^{3-9}$ chemical sensors, ${ }^{10}$ organic solar cells, ${ }^{11}$ as well as transparent electrodes in photovoltaic devices. ${ }^{12,13}$ However, the electrical conductivity and field effect mobility values for RGO sheets are much inferior to that of pristine graphene. This has been attributed to a large amount of disorder present in the RGO sheets. Disorder in RGO sheets have mostly been characterized with temperature dependence of resistivity where variable range hopping and activated hopping have been observed. ${ }^{6-8}$ Such a study does not provide detailed information about the nature and density of charge traps in the system. A detailed knowledge of charge conduction and quantitative information about charge traps is important for further optimization of reduction techniques and synthetic strategies for increasing conductivity and mobility of RGO toward pristine graphene. Similar strategies have been successfully used for passivating defects in silicon to fabricate high quality complementary metal oxide semiconductor circuits.

In this paper we present measurements and analysis of temperature dependent current density $(J)$-voltage $(V)$ characteristics to elucidate on the nature and density of charge traps in the RGO sheets. The GO sheets suspended in water were reduced chemically in the solution and then assembled between prefabricated gold source and drain electrodes via ac dielectrophoresis (DEP). We show that the $J$ - $V$ character-

\footnotetext{
a) Author to whom correspondence should be addressed. Electronic mail: saiful@mail.ucf.edu.
}

istic of the RGO devices measured at different temperatures ( 295 to $77 \mathrm{~K}$ ) follow power law behavior, $J \infty V^{m}$. At low bias, the conduction is Ohmic with $m=1$, while at higher bias voltages $m$ increases from 2 to 3 with reducing temperature signifying space charge limited conduction (SCLC) with a transition from trap free (TF-SCLC) regime at room temperature to exponentially distributed trap (EDT-SCLC) regime at low temperatures. We estimate an average trap density of $1.75 \times 10^{16} \mathrm{~cm}^{-3}$ for our samples.

RGO sheets were synthesized through a chemical reduction of GO in solution prepared by modified Hummers method. ${ }^{14}$ Oxidized graphite in water was ultrasonicated to achieve GO monolayer sheets followed by centrifugation for $30 \mathrm{~min}$ at $3000 \mathrm{rpm}$ to remove any unexfoliated oxidized graphite. The $p \mathrm{H}$ of $\mathrm{GO}$ dispersion in water $(0.1 \mathrm{mg} / \mathrm{mL})$ was adjusted to 11 using a 5\% ammonia aqueous solution. $15 \mu \mathrm{L}$ of hydrazine solution $(35 \%$ in $\mathrm{N}, \mathrm{N}-$ dimethylformamide) was then added and the mixture was heated at $95-100{ }^{\circ} \mathrm{C}$ for $1 \mathrm{~h}$. X-ray photoelectron spectroscopy spectra show effective reduction in GO (see Ref. 15). Devices were fabricated on heavily doped silicon $(\mathrm{Si})$ substrates capped with a thermally grown $250 \mathrm{~nm}$ thick $\mathrm{SiO}_{2}$ layer. Source and drain electrode patterns with a channel length of $500 \mathrm{~nm}$ and width of $500 \mathrm{~nm}$ were defined by electron beam lithography and thermal deposition of $5 \mathrm{~nm} \mathrm{Cr}$ and $20 \mathrm{~nm} \mathrm{Au}$ followed by lift off.

The RGO sheets were assembled between the source and drain electrodes via DEP. Figure 1(a) shows a cartoon of the DEP setup. Details of the assembly were presented in our previous publication. ${ }^{9}$ In brief, a small drop of RGO solution was placed onto the electrodes pattern. An ac voltage of approximately $3 V_{P-P}$ at $1 \mathrm{MHz}$ was applied for $20-30 \mathrm{~s}$ after which the solution droplet was blown off by nitrogen gas. Figure 1(b) shows tapping-mode atomic force microscope (AFM) image of a representative devices with the height analysis shown in Fig. 1(c). It can be seen that the thickness varies from 2 to $8 \mathrm{~nm}$ in the channel indicating that up to eight layers of RGO sheets have been assembled. The devices were then annealed in $\mathrm{Ar} / \mathrm{H}_{2}$ gas at $200{ }^{\circ} \mathrm{C}$ for an 

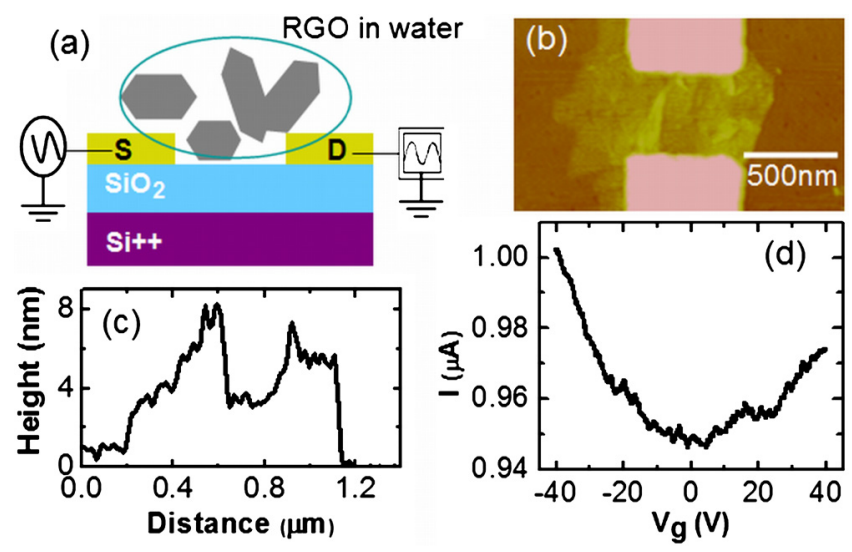

FIG. 1. (Color online) (a) Cartoon of DEP assembly setup. (b) Tappingmode AFM of a RGO device assembled via DEP. Scale bar represents 500 nm. (c) Height profile of the AFM image shown in Fig. 1(b). (d) Room temperature transfer characteristic of device A.

hour, and loaded into a variable temperature cryostat after bonding. Temperature dependent electronic transport measurements from 295 to $77 \mathrm{~K}$ were performed using a Keithley 2400 source-meter, and a current preamplifier (DL 1211) capable of measuring subpicoampere signal interfaced with LABVIEW program. A total of nine devices were investigated.

Figure 1(d) shows the room temperature transfer characteristics of a representative RGO device (device A) where the current $(I)$ is plotted as a function of gate voltage $\left(V_{g}\right)$ measured in vacuum. The gate voltage was scanned from -40 to $+40 \mathrm{~V}$ with a fixed source-drain voltage of $V=0.5 \mathrm{~V}$. The device shows an ambipolar FET behavior while. The room temperature hole mobility and the carrier concentration were calculated as $0.71 \mathrm{~cm}^{2} / \mathrm{V} \mathrm{s}$ and $12 \times 10^{12} \mathrm{~cm}^{-2}$, respectively. The FET properties are similar to other studies reported previously. ${ }^{3-9}$ However, recent report suggests that the mobility can be significantly higher when the size of RGO is big and reduction is done at very high temperature. ${ }^{16,17}$

Figures 2(a) and 2(b) show the $I-V$ characteristics at various temperatures measured from -5 to $5 \mathrm{~V}$ with $V_{g}=0$ for device A. The magnitude of the applied bias was limited to $5 \mathrm{~V}$ to avoid electrical breakdown of the RGO devices. The $I-V$ curves are highly symmetric at all temperatures. In addition, the $I-V$ curve became increasingly nonlinear with decreasing temperature. The nonlinearity in $I-V$ curves in disordered semiconductor systems has been explained using (i) Schottky barrier (SB) between metal electrode and semiconductor, (ii) Fowler-Nordheim (FN) tunneling, and (iii) SCLC. In general, the $I-V$ curve of a metal-semiconductormetal junction should be highly asymmetric if the SB is dominated. However, our $I-V$ curves are highly symmetric [see Fig. 2(b)] giving evidence that charge transport is not dominated by SB. Additionally, the attempt to fit our data with the SB was not successful (not shown here). FN tunneling can be ruled out as in FN model, ${ }^{18}$ the $I-V$ curves are independent of temperature while our $I-V$ curves are highly sensitive to temperature.

We now discuss SCLC mechanism. SCLC occurs in low mobility semiconductors when injected charge density exceeds the intrinsic free carrier density of the material. Analysis of the $J-V$ characteristics using the SCLC model is one of the experimental methods for the detection of charge trap states in disordered semiconductors. In the ab-
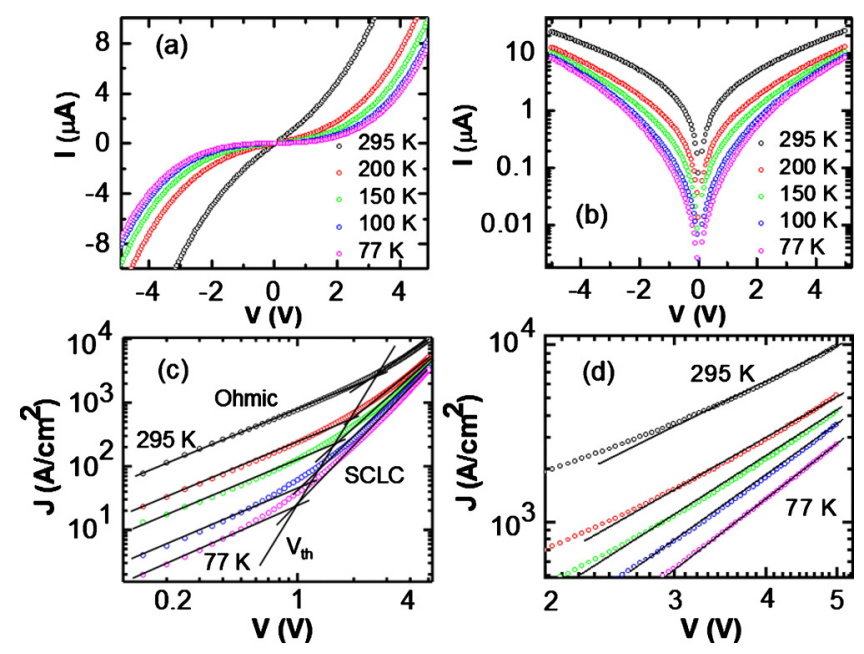

FIG. 2. (Color online) (a) Current-Voltage $(I-V)$ characteristics at different temperatures for device A. (b) $I$ - $V$ curves at different temperatures plotted in semi log scale to clearly show highly symmetric nature of the curves. (c) Current density $(J)$ vs $V$ plotted in a log-log scale for different temperatures. The symbols are the experimental points while the solid lines are fits to $J \propto V^{m}$. Two regions are separated by $V_{t h}$. For $V<V_{t h}, m=1$ and the conduction is Ohmic. $V>V_{t h}$ the conduction is due to SCLC with exponentially distributed trap states. (d) Expanded view of the SCLC regime. For clarity, $J$ at $77 \mathrm{~K}$ was divided by 1.2 .

sence of any trap states or when trap states do not dominate the transport, the $J-V$ characteristics are described using, $J=9 \varepsilon_{0} \varepsilon_{r} \mu V^{2} / 8 d^{3}$ where $\varepsilon_{0}$ is the permittivity of free space, $\varepsilon_{r}$ the dielectric constant of the RGO, $\mu$ is the charge carrier mobility, and $d$ is the spacing between electrodes. ${ }^{19}$ However, in presence of trap states that are exponentially distributed in energy, the $J-V$ relationship is given by $J=\left(\mu N_{v} / q^{l-1}\right)[(2 l+1) /(l+1)]^{1 / l}\left\{\left(\varepsilon \varepsilon_{0} / N_{t}\right)[l /(l\right.$ $+1)]\}^{l}\left(V^{l+1} / d^{2 l+1}\right)$ where, $l$ is an exponent and should be greater than $1, q$ is the electronic charge, $N_{v}$ is effective density of states, and $N_{t}$ is the trap density. ${ }^{20}$ By plotting $J$ and $V$ in $\log -\log$ scale one can determine the value of exponent $\mathrm{m}$. For trap free (TF-SCLC) regime, $m=2$ while for exponentially distributed trap (EDT-SCLC) regime $m=l$ $+1>2$.

Fig. 2(c) shows $J$ versus $V$ curves at different temperature in a log-log scale for sample A. The dotted symbols are the experimental data points and the solid lines are a fit to $J \propto V^{m}$. At low voltages, $m$ equals to 1 at all temperatures signifying Ohmic conduction. However, at higher voltages, $m$ deviates from $1 . V_{t h}$, the onset voltage where $\log J-\log V$ curves begin to inflect to higher slope region, shifts to lower voltage with decreasing temperature. For $V>V_{t h}, m=2$ at room temperature implying TF-SCLC regime. However, as the temperature is reduced, the value of $\mathrm{m}$ for $V>V_{t h}$ is increased to 2.3 at $200 \mathrm{~K}, 2.6$ at $150 \mathrm{~K}, 2.8$ at $100 \mathrm{~K}$, and 3 at $77 \mathrm{~K}$. The $J-V$ curves for higher values of $V$ are more clearly shown in Fig. 2(d). For these temperatures, the conduction is governed by traps that are exponentially distributed in energy (EDT-SCLC).

A transition from TF-SCLC regime at room temperature to EDT-SCLC regime at low temperature can occur for the following reason: At room temperature, the free career density is higher than the trap density. However, as the temperature is reduced, free career density is also reduced and the trap states started to dominate by localizing charge carriers and we enter EDT-SCLC regime with $m>2$. 


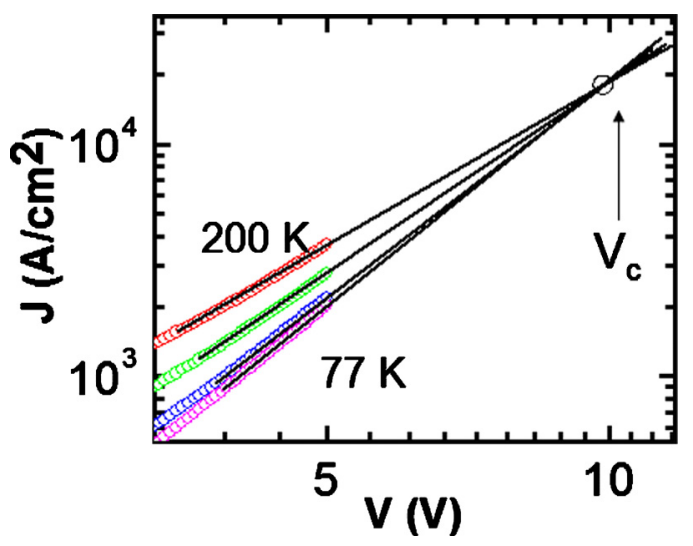

FIG. 3. (Color online) Extrapolation of the $J$ - $V$ curves at low temperatures plotted in $\log -\log$ scale for device A. From this plot, we determine the temperature independent crossover voltage $V_{c}=10 \mathrm{~V}$.

More quantitative information about traps can be obtained by extrapolating $J-V$ curves in voltage. If charge traps are distributed in energy, they will be gradually filled with increasing electric field at all temperatures and at a certain critical voltage, all traps will be filled. According to Kumar et $a ._{.}{ }^{21}$ this critical voltage is independent of temperature and is given by $V_{c}=q N_{t} d^{2} / 2 \varepsilon_{r} \varepsilon_{0}$. By extrapolating the $J$ - $V$ curves we can obtain values for $V_{c}$ and calculate trap density $N_{t}$. In Fig. 3, we extrapolate the $\log J$ and $\log V$ characteristic at higher bias voltages for all temperatures except room temperature. The $V_{c}=10 \mathrm{~V}$ was found for device A. In order to calculate the trap density from $V_{c}$, we need to know the dielectric constant $\left(\varepsilon_{r}\right)$ of chemically RGO. The value of $\varepsilon_{r}$ can be calculated by using; $\varepsilon_{r}=n^{2}-k^{2}$, where $n$ and $k$ are refractive index and extinction coefficient of RGO film, respectively. Using the values of $n$ and $k$ in the thermally reduced $\mathrm{GO}^{22}$ we calculate $\varepsilon_{r}=3.5$. Using this value, we obtain trap density $N_{t}=1.54 \times 10^{16} \mathrm{~cm}^{-3}$ for A.

Similar EDT-SCLC regime at low temperatures was observed in all nine samples that we have measured. In Ref. 15 (Table I), we summarize all measured RGO devices where we tabulate room temperature hole mobility, $V_{c}$ and $N_{t}$ values. All measured devices show similar trap density. The average trap density was about $1.75 \times 10^{16} \mathrm{~cm}^{-3}$.

An exponential distribution of trap in energies is expected for the traps originating from surface defects and structural disorders. ${ }^{23}$ When GO is produced via oxidation, some $\mathrm{C}-\mathrm{C}$ bonds can get broken creating vacancies. ${ }^{24}$ During the reduction process, carbon can also get removed from the GO backbone. ${ }^{25}$ Although, this has been theoretically shown for thermal reduction cases, however, such study for chemical reduction cases are yet to be done. In addition, line defects such as wrinkles and folding of RGO sheet also create defects. ${ }^{2}$ Because of the presence of the large number of trap states, charge in the gate would induce charge in the traps rather than free charge carrier in the semiconducting RGO sheets causing a low mobility in these sheets. Our study suggests that a large number of trap states exists in RGO and call for more theoretical and experimental investigations to understand how the trap states are created and how can they be minimized.

In conclusion, we elucidated on the low mobility and density of charge traps in RGO sheets by measuring and analyzing temperature dependent current-voltage characteris- tics. The GO sheets suspended in water were reduced chemically in the solution and then assembled between prefabricated gold source and drain electrodes via ac DEP. We show that at low bias voltage the conduction is Ohmic while at high bias voltage the conduction becomes SCLC. At room temperature, the conduction is governed by trap free SCLC while at a lower temperature it is dominated by traps that are exponentially distributed in energy. We estimated an average trap density of $1.75 \times 10^{16} \mathrm{~cm}^{-3}$. Quantitative studies of charge traps using SCLC presented in this work will facilitate further development of strategies for the chemical modification of RGO surfaces to passivate traps and thereby improve the carrier transport by providing quantifying evidences of the developed strategies of passivating defects in order to fabricate high quality solution processed graphene devices.

This work has been partially supported by U.S. NSF under Grant No. ECCS 0748091 to S.I.K. and under Grant No. DMR 0746499 to L.Z.

${ }^{1}$ S. Stankovich, D. A. Dikin, G. H. B. Dommett, K. M. Kohlhaas, E. J. Zimney, E. A. Stach, R. D. Piner, S. T. Nguyen, and R. S. Ruoff, Nature (London) 442, 282 (2006).

${ }^{2}$ H. C. Schniepp, J. L. Li, M. J. McAllister, H. Sai, M. Herrera-Alonso, D. H. Adamson, R. K. Prud'homme, R. Car, D. A. Saville, and I. A. Aksay, J. Phys. Chem. B 110, 8535 (2006).

${ }^{3}$ S. Gilje, S. Han, M. Wang, K. L. Wang, and R. B. Kaner, Nano Lett. 7, 3394 (2007).

${ }^{4}$ I. Jung, D. A. Dikin, R. D. Piner, and R. S. Ruoff, Nano Lett. 8, 4283 (2008).

${ }^{5}$ G. Eda, G. Fanchini, and M. Chhowalla, Nat. Nanotechnol. 3, 270 (2008).

${ }^{6}$ C. Gómez-Navarro, R. T. Weitz, A. M. Bittner, M. Scolari, A. Mews, M. Burghard, and K. Kern, Nano Lett. 7, 3499 (2007).

${ }^{7}$ A. B. Kaiser, C. Gómez-Navarro, R. S. Sundaram, M. Burghard, and K. Kern, Nano Lett. 9, 1787 (2009).

${ }^{8}$ G. Eda, C. Mattevi, H. Yamaguchi, H. Kim, and M. Chhowalla, J. Phys. Chem. C 113, 15768 (2009).

${ }^{9}$ D. Joung, A. Chunder, L. Zhai, and S. I. Khondaker, Nanotechnology 21, 165202 (2010).

${ }^{10}$ G. Lu, L. E. Ocola, and J. Chen, Appl. Phys. Lett. 94, 083111 (2009).

${ }^{11}$ Q. Liu, Z. Liu, X. Zhang, N. Zhang, L. Yang, S. Yin, and Y. Chen, Appl. Phys. Lett. 92, 223303 (2008).

${ }^{12}$ X. Wang, L. Zhi, and K. Mullen, Nano Lett. 8, 323 (2008).

${ }^{13}$ J. Wu, H. A. Becerril, Z. Bao, Z. Liu, Y. Chen, and P. Peumans, Appl Phys. Lett. 92, 263302 (2008).

${ }^{14}$ M. Hirata, T. Gotou, S. Horiuchi, M. Fujiwara, and M. Ohba, Carbon 42 2929 (2004)

${ }^{15}$ See supplementary material at http://dx.doi.org/10.1063/1.3484956 for supplementary materials containing XPS data of GO and RGO to check effectiveness of reduction process, and summary of all measured RGO devices.

${ }^{16}$ S. Wang, P. K. Ang, Z. Wang, A. L. L. Tang, J. T. L. Thong, and K. P. Loh, Nano Lett. 10, 92 (2010).

${ }^{17}$ H. Yamaguchi, G. Eda, C. Mattevi, H. Kim, and M. Chhowalla, ACS Nano 4, 524 (2010).

${ }^{18}$ S. M. Sze, Physics of Semiconductor Devices, 2nd ed. (Wiley, New York, 1981).

${ }^{19}$ N. F. Mott and R. W. Gurney, Electronic Processes in Ionic Crystal (Dover, New York, 1964).

${ }^{20}$ P. Mark and W. Helfrich, J. Appl. Phys. 33, 205 (1962).

${ }^{21}$ V. Kumar, S. C. Jain, A. K. Kapoor, J. Poortmans, and R. Mertens, J. Appl. Phys. 94, 1283 (2003).

${ }^{22}$ I. Jung, M. Vaupel, M. Pelton, R. Piner, D. A. Dikin, S. Stankovich, J. An, and R. S. Ruoff, J. Phys. Chem. C 112, 8499 (2008).

${ }^{23}$ S. Baidyaroy and P. Mark, Surf. Sci. 30, 53 (1972).

${ }^{24}$ W. Zhang, V. Carravetta, Z. Li, Y. Luo, and J. Yang, J. Chem. Phys. 131, 244505 (2009).

${ }^{25}$ A. Bagri, C. Mattevi, M. Acik, Y. J. Chabal, M. Chhowalla, and V. B. Shenoy, Nat. Chem. 2, 581 (2010). 